\title{
Techniques to perform a laparoscopic right adrenalectomy for metastases abutting the liver, renal vein, and posterior vena cava
}

\author{
Ajay V. Maker ${ }^{1} \cdot$ Ryan Carr ${ }^{1}$
}

Received: 2 January 2015/Accepted: 8 April 2015/Published online: 3 July 2015

(C) Springer Science+Business Media New York 2015

\begin{abstract}
Introduction Introduction Laparoscopic adrenalectomy (LA) may be associated with reduction in postoperative pain, morbidity, length of stay, and cost compared to open adrenalectomy, and, as a result, has become a preferred approach for benign tumors [1]. Though recent series have reported safety and oncologic non-inferiority with a minimally invasive approach to malignant and metastatic adrenal tumors, relative contraindications to LA include malignancy, large size, and invasion or abutment of vital structures [2].

Methods/Results We demonstrate techniques to perform LA in an obese patient with a large $([6 \mathrm{~cm})$ solitary breast metastasis to the right adrenal gland that was adherent to the liver, inferior vena cava (IVC), and right renal vein. The tumor was growing behind the infrahepatic IVC and was abutting the confluence of the renal vein/IVC. The patient was monitored on systemic treatment with no new sites of disease and after achieving a maximal response to neoadjuvant treatment. Laparoscopic techniques to safely approach adrenal tumors adherent to the liver, IVC, and renal vein are stepwise demonstrated, including appropriate
\end{abstract}

patient positioning and port site selection. The tumor was excised with negative margins, and the patient was discharged on postoperative day 1 with minimal pain medication and tolerating a regular diet.

Conclusion Though malignancy is a relative contraindication to LA, even large right-sided adrenal tumors abutting the liver and growing behind the IVC can be safely approached while adhering to oncologic principles, as is demonstrated in this video.

Keywords Adrenal $\cdot$ Surgical $\cdot$ Technical $\cdot$ Cancer . Hepato $\cdot$ Metastases $\cdot$ Minimally invasive .

Adrenalectomy $\cdot$ Laparoscopic

\section{References}

1. Bradley CT, Strong VE (2014) Surgical management of adrenal metastases. J Surg Oncol 109:31-35

2. Strong VE, D'Angelica M, Tang L, Prete F, Gonen M, Coit D, Touijer KA, Fong Y, Brennan MF (2007) Laparoscopic adrenalectomy for isolated adrenal metastasis. Ann Surg Oncol 14:33923400

Presented, in part, at the American College of Surgeons Clinical Congress, San Francisco, CA, October, 2014.

Electronic supplementary material The online version of this article (doi:10.1007/s00464-015-4311-x) contains supplementary material, which is available to authorized users.

\footnotetext{
Ajay V. Maker

amaker@uic.edu

1 Division of Surgical Oncology, Department of Surgery, University of Illinois at Chicago, 835 S. Wolcott Ave., MC790, Chicago, IL 60612, USA
} 